\title{
Seven Reasons To Be Concerned About the Use of the New Preim- plantation Genetic Screening (PGS)
}

\author{
Jose G. Franco Jr
}

President of SBRA - Brazilian Society of Assisted Reproduction/Scientific Director of Center for Human Reproduction Prof. Franco Jr, Ribeirao Preto, SP, Brazil

In theory, PGS should improve embryo implantation and reduce miscarriage rates. This approach can be indicated for women of advanced maternal age, and those with repeated implantation failure, and to select the best embryo to transfer in good prognosis patients.

\section{Do not forget evidence-based medicine}

In the few randomized control trials (RCT) published about the new way of running the PGS (PGS-new), the lessons of reproductive evidence-based medicine are often ignored. The " $n$ " sample is always lower than required and the concept of intention-to-treat analysis (ITT) is forgotten. It should be remembered that all randomized patient need to be analyzed independently of protocol deviations, withdrawal, and anything that happens after randomization. The patients should not be randomized by the number of embryos. Usually, in the new-PGS there are a minimum number of viable blastocysts as a rule to initiate patient randomization.

\section{An adverse past and an uncertain future}

In 2007, Mastenbroek et al. published a RCT $(n=206$ patients in PGS and $n=202$ in the control group; ITT present) from a study in which the indication for PGS was advanced maternal age. PGS (day 3 biopsy + fluorescence in situ-hybridization-FISH) did not increase, but instead, it significantly reduced the rates of ongoing pregnancies and live births (PGS $=24 \%$; Control $=35 \%$ ). This was the first time in which the use of PGS was criticized. On this occasion, many who advocated PGS (Munné et al., 2007) did not accept the results presented by Mastenbroek et al.: "We believe that study design does not reflect usual clinical practice in pre-implantation genetic screening centers. A minimum of 6 to 8 embryos has thus been recommended when using PGS". From a clinical point of view, it is difficult to understand how patients at a mean age $\geq 38$ years (advanced age), routinely produced a total minimum of 6 embryos to be submitted to PGS as the method for embryo selection. This would be like denying proven aspects such as the reduction in ovarian reserve and the response to stimulation with aging. After a few years, the advocates of PGS accepted the criticism of new RCT about the PGS efficacy. Thus, they started to assign the burden of negative outcomes to the imperfect FISH technique in the assessment of chromosome abnormalities, and to the fact that the biopsy carried out on the third day (embryo cleavage stage) would carry a high frequency of mosaicism. Thus the PGS-new started with the embryonic biopsy on the 5th or 6th day (lower mosaicism), and the use of new techniques of chromosome assessment (Comparative genomic hybridization-CGH; Array-CGH-aCGH; Real-time quantitative polymerase chain-reaction-qPCR; Next generation sequencing-NGS), safer in establishing chromosomal abnormalities, in addition to enabling the analysis of 24 chromosomes.

At the moment, despite these new arguments, there are no proper RCT publications (ideal " $n$ " + ITT) with PGSnew in populations of advanced age.

\section{The trophectoderm is an area of chromosomal variability}

The trophectoderm blastocyst biopsy is performed in the PGS-new. Mosaicism is an eternal problem (Taylor et al., 2014). Cells (trophectoderm) not destined to become the fetus may not be a true representation of the fetus itself, but rather of extraembryonic tissue (cytotrophoblast, chorion, placenta, extraembryonic mesoderm). The aneuploidy rate can be around $70 \%$ in day 3 versus approximately $20 \%-50 \%$ in the blastocyst, and then there could be a significant degree of embryo self-correction. Yang et al. (2012) described $35.6 \%$ of aneuploid blastocysts with single chromosome loss (monosomy). Recently, Gleicher et al. (2015) associated the normal live birth of 3 patients from a total of 5 after embryo transfer of PGS-new with the diagnosis of monosomy (Case 1: 2 embryos with 13,15/16,18 monosomy; Cases 2 and 3: 1 embryo with 21 monosomy).

\section{Data are missing for several indications}

In 2008, Blockeel et al. employed PSG in a randomized and prospective way to patients with repeated implantation failure; they showed a significant difference in live birth in favor of the control group (39\%/ $\mathrm{n}=67)$ versus PGS $(21 \% / \mathrm{n}=72)$ (RD: $0.03,95 \% \mathrm{CI}$ : -0.33 to -0.03$)$. At the moment, there is no proper RCT (ideal sample " $n$ " + ITT) was carried out with the PGSnew in populations with repeated implantation failure.

\section{Indicating PGS-new for infrequent populations}

In 2011, 3 RCTs were published about the use of PGS in patients labeled as good prognosis (Staessen et al., 2008; Jansen et al., 2008; Meyer et al., 2009). Back then, they did not find significant differences in terms of live birth rate among patients submitted to PGS, or not. In 2015, Dahdouh et al. found 3 RCTs (Yang et al., 2012; Forman et al., 2013; Scott et al., 2013) reporting the use of PGS-new in good prognosis patients. In the RCT published by Yang et al. (2012), 112 patients were randomized ( $n=56 /$ PGS-new; $n=56 /$ PGS Control, ITT present) with mean age of $31.2 \pm 2.5$ years/PGS-new and $31.5 \pm 2.7 /$ Control, they used $\mathrm{aCGH}$, with a mean of $19.5 \pm 8.2$ /PGS-new and 19.3 $\pm 8.1 /$ Control oocytes collect-

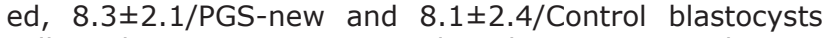
collected. PGS-new improves clinical pregnancy and ongoing pregnancy rates (PGS-new: $67.8 \%$ versus $35.7 \%$ for Controls).

The number of oocytes collected was high in both groups, which limits the value of PGS-new employment, even in patients considered of good prognosis. In our service, $<5 \%$ of the patients had $\geq 19$ egg-cells collected. It is most interesting that no cases of ovary hyperstimulation were observed in this population.

On the other hand, the number of cryopreserved embryos and the results of subsequent thawing cycles were 
not reported. This is a potential cause of bias as it can be expected that more embryos are cryopreserved among controls, and these embryos are likely to contribute to the cumulative pregnancy rate (fresh + thawing cycles). (Mastenbroek \& Repping, 2014).

In the RCT by Forman et al. (2013) 175 patients were randomized (ITT absent). Randomization occurred when at least two embryos had developed sufficiently to allow for blastocyst-stage biopsy (before randomization, 11 patients were excluded because no blastocyst was developed). In this RCT the comparison was between elective single embryo transfer of an embryo that had been screened by PGS-new against two morphologically selected. Mean age was $35.1 \pm 3.9$ years/PGS-new and $34.5 \pm 4.0$ /Control, they employed the qPCR, and the mean number of oocytes col-

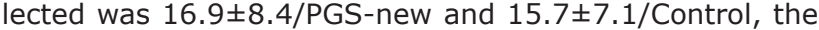
number of blastocysts obtained was 5.8 $\pm 3.6 /$ PGS-new and $5.3 \pm 3.0 /$ Control. Similar pregnancy rates were observed between the two groups.

The original intent of this study was to improve IVF pregnancy rates. As this failed, their original intent was replaced by the listed secondary goal of this study: reduction of twin pregnancies at elective single transfer (Gleicher et al., 2014).

In the RCT from Scott et al. (2013) randomization was carried out after the selection of patients with at least two blastocysts (ITT absent). The mean age was $32.2 \pm 0.5$ years/PGS-new and $32.4 \pm 0.5 /$ Control. They employed the qPCR, and the mean numbers of oocytes

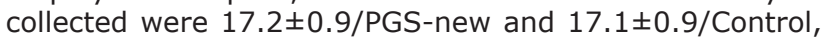
the number of blastocysts obtained was $8.0 \pm 0.7 /$ PGSnew and $7.9 \pm 0.7 /$ Control. The results of the PGS-new showed improvements in implantation and delivery rates. Once again, they did not evaluate the cumulative results of clinical pregnancy and delivery rates by including the analysis of thawing cycles. The sample " $n$ " was lower that what would be necessary to validate the conclusions as to variables: clinical pregnancy and delivery rates. In short, the few RCTs about PGS-new are not convincing, restricted to special populations (a mean of 16 egg-cells collected and 6 blastocysts produced) and infrequent in the universe of patients who require ART.

\section{Concerns about extended culture to the blastocyst stage}

Blastocyst stage in vitro cultures have also been recently reported to be associated with two significant additional potential risks: 1- prolonged embryo culture has been related to significant epigenetic changes (Lonergan et al., 2003; Calle et al., 2012); 2- blastocyst stage culture are associated with increased risk of premature delivery in comparison to embryos transferred on days 2 or 3 (Maheshwari et al., 2013; Dar et al., 2014).

\section{Non-maleficence}

In 2008, the American Society of Assisted Reproductive Medicine (ASRM), the European Society for Human Reproduction and Embryology (ESHRE) and the British Fertility Society declared that PGS (day 3 biopsy + FISH technique) is ineffective in improving IVF pregnancy rates and reducing miscarriage. Seven years later, these societies have not yet settled for or against PGS-new (day 5-6 biopsy + CGH or qPCR or NGS), as well as the Brazilian Society of Assisted Reproduction (SBRA). Today there is no data supported by evidence-based medicine to release the routine use of PGS-new.

\section{REFERENCES}

Blockeel C, Schutyser V, De Vos A, Verpoest W, De Vos M, Staessen C, Haentjens P, Van der Elst J, Devroey P. Prospectively randomized controlled trial of PGS in IVF/ICSI patients with poor implantation. Reprod Biomed Online. 2008; 17: 848-54.

Calle A, Fernandez-Gonzalez $R$, Ramos-Ibeas $P$, Laguna-Barraza R, Perez-Cerezales $S$, Bermejo-Alvarez $P$, Ramirez MA, Gutierrez-Adan A. Long-term and transgenerational effects of in vitro culture on mouse embryos. Theriogenology. 2012:77:785-93.

Dahdouh EM, Balayla J, Garcia-Velasco JA. Impact of blastocyst biopsy and comprehensive chromosome screening technology on preimplantation genetic screening: a systematic review of randomized controlled trials. Reprod Biomed Online. 2015;30:281-89.

Dar S, Lazer T, Shah PS, Librach CL. Neonatal outcomes among singleton births after blastocyst versus cleavage stage embryo transfer: a systematic review and meta-analysis. Hum. Reprod. Update 2014;20:439-48.

Forman EJ, Hong KH, Ferry KM, Tao X, Taylor D, Levy B, Treff NR, Scott RT Jr. In vitro fertilization with single euploid blastocyst transfer: a randomized controlled trial. Fertil Steril. 2013; 100: 100-7.

Gleicher N, Kushnir V, Barad DH. Preimplantation genetic sreening (PGS) still in search of a clinical application: a systematic review. Reprod Biol Endocrinol. 2014; $12: 22$.

Gleicher N, Vidali A, Braverman J, Kushnir VA, Albertini DF, Barad DH. Further evidence against use of PGS in poor prognosis patients: report of normal births after transfer of embryos reported as aneuploidy. Fertil Steril. 2015; 104: e59.

Jansen RP, Bowmann MC, de Boer KA, Leigh DA, Lieberman D, McArthur SJ. What next for preimplantation genetic screening (PGS)? Experience with blastocyst biopsy and testing for aneuploidy. Hum Reprod. 2008;23:1476-8.

Lonergan P, Rizos D, Gutierrez-Adan A, Fair T, Boland MP. Effect of culture environment on embryo quality and gene expression-experience from animal studies. Reprod Biomed Online. 2003;7:657-63.

Mastenbroek S, Twist M, van Echten-Arends J, Sikkema-Raddatz B, Korevaar JC, Verhoeve HR, Vogel NE, Arts EG, de Vris JW, Bossuyt PM, Buys CH, Heineman MJ, Repping $S$, van der Veen $F$. In vitro fertilization with preimplantation genetic screening. N Engl J Med 2007;357:9-17.

Mastenbroek S, Repping S. Preimplantation genetic screening: back to the future. Hum Reprod. $2014 ; 29: 1846-50$.

Meyer LR, Klipstein S, Hazlett WD, Nasta T, Mangan P, Karande VC. A prospective randomized controlled trial of preimplantation genetic screnning in the good prognosis patients. Fertil Steril. 2009;91:1731-8.

Maheshwari A, Kalampokas T, Davidson J, Bhattacharya S. Obstetric and perinatal outcomes in singleton pregnancies resulting from the transfer of blastocyst-stage versus 
cleavage-stage embryos generated through in vitro fertilization treatment: a systematic review and meta-analysis. Fertil Steril 2013;100:1615-21.

Munné S, Cohen J, Simpson JL. In vitro fertilization with preimplantation genetic screening. N Engl J Med. 2007; 357:1769-70.

Staessen C, Verpoest W, Donoso P, Haentjens P, Van der
Elst J, Liebaers I, Devroey P. Preimplantation genetic screening does not improve delivery rate in couples under age of 36 following single-embryo transfer. Hum Reprod. $2008 ; 23: 2818-25$.

Taylor TH, Gitlin SA, Patrick JL, Crain JL, Wilson JM, Griffin DK. The origin, mechanisms, incidence and clinical consequences of chromosomal mosaicism in humans. Hum Reprod Update. 2014;20:571-81. 